\section{Drug delivery coalesces}

By creating chimeric proteins that contain the coding sequence for a conditional aggregation domain (CAD) fused to a proinsulin, a collaboration of industrial and acadmic scientists has come up with a protein delivery system that responds to exogenous treatment with a small molecule (Science 287, 826-830, 2000). When expressed in cells, the CAD fusions accumulate in the endoplasmic reticulum and form large aggregates. A small-molecule that binds to the CADs breaks up the aggregates, allowing the stored proteins to be secreted rapidly. To prove the concept, the team injected fibroblasts expressing a CAD-proinsulin fusion protein into the muscles of a mouse model of hyperglycemia. When the mice received the smallmolecule orally, cells rapidly secreted therapeutic quantities of the protein and transiently corrected the animals' serum glucose levels. Senior author Tim Clackson, a researcher at ARIAD Gene Therapeutics (Cambridge, MA), says that "the applications go beyond insulin to any protein or peptide that needs to be delivered in short bursts. We are especially interested in delivery of endorphins for management of acute pain, where rapid delivery is essential."

\section{Hot plants}

In a study with broad implications for agriculture, researchers at Kyushu University in Japan have produced transgenic tobacco plants that can sustain photosynthesis when the temperatures soar (Science 287, 476-479, 2000). Taking a cue from desert plants that reduce synthesis of trienoic fatty acids in chloroplasts at high temperatures, the researchers produced two transgenic tobacco lines containing an additional chloroplast-specific $\Delta$-3 desaturase gene from Arabidopsis thaliana. Cosuppression led to a reduction of $\Delta-3$ desaturase gene expression in these plants and a concomitant reduction in trienoic fatty acids. As a result, the transgenic plants had a higher photosynthetic activity at growing temperatures of $40^{\circ} \mathrm{C}$ (which greatly diminishes photosynthesis in wild-type plants) than at $25^{\circ} \mathrm{C}$. The scientists suggest that the use of endogenous genes avoided unexpected deleterious effects sometimes associated with gene manipulation using exogenous DNA. "This study may open the door for the use of gene manipulation techniques to breed new crops or trees that can adapt to inevitable changes in the global climate," says Koh Iba, a plant physiologist on the research team. "For example, it may be interesting to improve the adaptability of trees in [colder environments]...to a hightemperature environment."

\title{
Illuminating cancer models
}

A new system for studying tumor growth and metastasis using the popular green fluorescent protein (GFP) tag is being adapted as a possible platform for testing anti-angiogenic compounds. The basic approach, described recently in the Proc. Natl. Acad. Sci. USA (97, 1206-1211, 2000), employs rodent and human tumor cell lines that express high levels of GFP. When injected into appropriate mouse strains, the cells can be traced in real time using a noninvasive whole-body imaging technique. In the initial tests of this concept, researchers were able to observe the growth of primary tumors and metastases in vivo. According to Andrew Perry, president of AntiCancer A/C's (San Diego, CA) diagnostic division, at the March meeting of the American Association of Cancer Research in New Orleans, LA, scientists from the company will describe additional experiments that they say demonstrate the model's utility for studying angiogenesis in tumors. Perry said in a statement that "until now research has been significantly impeded through lack of a convenient and relevant animal model of angiogenesis. We anticipate our new product will greatly enhance the research."

\section{Solid-state DNA computer}

A team of scientists at the University of Wisconsin, Madison has shown that DNA computing is possible on a gold-plated chip no bigger than a ticket stub (Nature 403, 175-179, 2000.) By encoding immobilized DNA strands to represent all possible solutions of a logical problem and serially introducing complements of the strands as probes that meet the criteria of the solution, they were able to create steps equivalent to the Boolean functions "AND" and "OR." After the probes hybridized on the chip, any single strands (representing "wrong answers") were destroyed by addition of an enzyme. The remaining DNA was stripped of probe and the cycle repeated until all conditions were fulfilled. By PCR amplifying the remaining DNA and reading it on the fluorescent array, the scientists found the correct solution. "This is the culmination of three subprojects. . In this paper, we put all the little pieces together and demonstrate a calculation," says Robert Corn, professor of chemistry at the University of Wisconsin, Madison. Currently, research is underway to reduce binding and reading error, and create an "append" step, which could function as the chemical "IF". DNA computing has the potential to co-process an almost infinite number of variables simultaneously. Any additional variable in a logic problem thus requires a single additional step rather than increasing calculations exponentially, as in conventional computing.

\section{Spinal cord rejuvenation}

In an approach that might someday be used to treat traumatic nerve damage, researchers have used neurotrophic factors to regenerate sensory axons into the spinal cords of adult rats (Nature 403, 312-316, 2000). "These experiments relate to a specific type of spinal cord injury where the nerve roots of the brachial plexus are damaged or torn away from the spinal cord," says first author Matt Ramer, a researcher at Guy's, King's, and St. Thomas' School of Biomedical Sciences (London, UK). Such injuries, which cause incurable paralysis, are seen in motorcycle crash victims and can occur in infants as a result of complications during childbirth. In rats given a similar injury, the scientists found that injection of the neurotrophic factors nerve growth factor, neurotrophin-3, or glialderived neutrotrophic factor into the cerebrospinal fluid caused sensory neurons to regenerate into the spinal cord. Behavioral and electrophysiological analysis showed that the regenerated axons are apparently functional. Though the initial results are promising and some neurotrophic factors have already been approved for use in humans, Ramer concedes that the few clinical trials to test these factors for other indications have all failed: "Whether this is due to delivery problems associated with these proteins, the doses employed or other factors is not yet known."

Research News Briefs written by Alan Dove and Keely Savoie. 